\title{
DERIVATIVES OF ENTIRE FUNCTIONS AND A QUESTION OF PÓLYA
}

\author{
BY \\ SIMON HELLERSTEIN(1) AND JACK WILLIAMSON
}

ABSTRACT. An old question of Pólya asks whether an entire function $f$ which has, along with each of its derivatives, only real zeros must be of the form

$$
f(z)=z^{m} e^{-a z^{2}+b z+c} \prod_{n}\left(1-\frac{z}{z_{n}}\right) e^{z / z_{n}}
$$

where $a>0, b$ and the $z_{n}$ are real, and $\sum_{n} z_{n}^{-2}<\infty$. This note answers this question (essentially in the affirmative) if $f$ is of finite order; indeed, it is established that if $f, f^{\prime}$, and $f^{\prime \prime}$ have only real zeros ( $f$ of finite order), then either $f$ has the above form or $f$ has one of the forms

$$
f(z)=a e^{b z}, \quad f(z)=a\left(e^{i c z}-e^{i d}\right)
$$

where $a, b, c$, and $d$ are constants, $b$ complex, $c$ and $d$ real.

Introduction(2). This note is concerned with real entire functions $f$ and the influence the growth of $f$ has on the distribution of the zeros of $f^{\prime}$ and $f^{\prime \prime}$. Recall that a real entire function is one which assumes real values on the real axis. In order to state concisely the background to our results, as well as the results themselves, we introduce the following notation: For each integer $p \geqslant 0$, denote by $V_{2 p}$ the class of entire functions of the form

$$
f(z)=\exp \left(-a z^{2 p+2}\right) g(z)
$$

where $a \geqslant 0$ and $g(z)$ is a constant multiple of a real entire function of genus $\leqslant 2 p+1$ with only real zeros. That is, $g$ is of the form

$$
g(z)=c z^{m} e^{Q(z)} \prod_{n}\left(1-\frac{z}{z_{n}}\right) \exp \left(\frac{z}{z_{n}}+\cdots+\frac{1}{q}\left(\frac{z}{z_{n}}\right)^{q}\right)
$$

Presented to the Society, April 11, 1975 under the title Derivatives of entire functions and a conjecture of Pollya; received by the editors May 15, 1975 and, in revised form, November 24, 1975.

AMS (MOS) subject classifications (1970). Primary 30A66; Secondary 30A68, 30A70.

Key words and phrases. Real entire function, finite order, finite genus, derivative.

(1) Supported in part by NSF Grant GP 21340.

(2) This introduction is a somewhat expanded version of the authors' announcement [8] of the results of this paper.

- American Mathematical Society 1977 
where $c$ is a constant, $Q(z)$ is a real polynomial of degree $\leqslant 2 p+1, z_{n}$ are real, $\Sigma_{n}\left|z_{n}\right|^{-q-1}<\infty$, and $q \leqslant 2 p+1$. Now set $U_{0}=V_{0}$, and for $p \geqslant 1$, set $U_{2 p}=V_{2 p}-V_{2 p-2}\left({ }^{3}\right)$.

The class $U_{0}$ is of particular interest, for a classical theorem of Laguerre [9] and Pólya [12] asserts that $f \in U_{0}$ if and only if it can be uniformly approximated on discs about the origin by a sequence of polynomials with only real zeros.

An immediate consequence of this fact is the following

THEOREM A. The class $U_{0}$ is closed under differentiation; that is, if $f \in U_{0}$, then $f^{(n)} \in U_{0}, n=1,2, \ldots$ In particular, $f \in U_{0}$ implies $f^{(n)}$ has only real zeros $n=1,2, \ldots$.

In 1914, Pólya [13] asked whether this theorem has a converse: Is $U_{0}$ the only class of entire functions closed under differentiation? That is, if an entire function $f$ and each of its derivatives have only real zeros, is $f \in U_{0}$ ?

Pólya showed [13] (a detailed proof will be found in [14]) that if one considers entire functions of the form $P(z) e^{Q(z)}$, where $P(z)$ and $Q(z)$ are polynomials, then aside from functions of the form $a e^{b z}$ ( $a$ and $b$ constants, $b$ complex), the answer is yes. Moreover, in [14] he conjectured that aside from a function $f$ of the form

$$
f(z)=a e^{b z} \text { or } f(z)=a\left(e^{i c z}-e^{i d}\right)
$$

where $a, b, c$, and $d$ are constants, $b$ complex, $c$ and $d$ real, only a function $f \in U_{0}$ has the property that it and each of its derivatives have only real zeros.

Alander subsequently devoted a series of papers to Pólya's question. In [1] and [2] he showed that if one restricts oneself to the classes $U_{2 p}, p=0,1,2$, then only the class $U_{0}$ is closed under differentiation, while in [3] he purports to have extended this result to arbitrary $p$. In a famous survey article on zeros of successive derivatives [15], Pólya refers to Ålander's papers [1] and [2], but not to his more general result [3]. The first author of this paper while a graduate student under the direction of A. Edrei brought this curious omission to the latter's attention. In response to Edrei's subsequent query, Pólya replied in a letter that he was aware of Ålander's more general "proof" but was never convinced by it nor could he show that it was fallacious!

Allander's "proof" involves a study of level curves of harmonic functions associated with functions in $U_{2 p}$. Avoiding such geometric considerations and using instead direct, analytic arguments, we prove the following stronger version of Ålander's "theorem."

(3) This classification of (constant multiples of) real entire functions was introduced by Ålander [3]. 
THEOREM 1. Let $f \in U_{2 p}$. If $f^{\prime}$ has only real zeros, then $f^{\prime \prime}$ has exactly $2 p$ complex (i.e., nonreal) zeros.

This result also partially affirms a long standing conjecture of Wiman $[3, \mathrm{p}$. 6]: If $f \in U_{2 p}$, then $f^{\prime \prime}$ has at least $2 p$ complex zeros.

An immediate consequence of Theorem 1 is

COROLlary 1. Let $f$ be a (constant multiple of a) real entire function of finite order. If $f, f^{\prime}$, and $f^{\prime \prime}$ have only real zeros, then $f \in U_{0}$.

For arbitrary entire functions of finite order, we verify Pólya's conjecture by our

THEOREM 2. Let $f$ be an entire function of finite order. If $f, f^{\prime}$, and $f^{\prime \prime}$ have only real zeros, then either $f \in U_{0}$ or $f$ has one of the forms

$$
f(z)=a e^{b z}, \quad f(z)=a\left(e^{i c z}-e^{i d}\right)
$$

where $a, b, c$, and $d$ are constants, $b$ complex, $c$ and $d$ real.

For arbitrary entire functions, the requirement of Theorem 2 that $f$ be of finite order is essential, as the example $\exp \left(e^{i z}\right)$ due to Edrei [7] shows. On the other hand, for (constant multiples of) real entire functions of infinite order the authors conjecture the following analog of Theorem 1:

HYPOTHETICAL THEOREM. Let $f$ be a (constant multiple of a) real entire function of infinite order with only real zeros. If $f^{\prime}$ has only real zeros, then $f^{\prime \prime}$ has an infinite number of complex zeros.

B. Ja. Levin and I. V. Ostrovskii [11] have proved this theorem for functions for which (roughly speaking) $M(r, f)=\max _{|z|=r}|f(z)|$ grows asymptotically faster than $\exp (\exp r)$. Since Theorem 1 together with an affirmative answer to the Hypothetical Theorem would completely answer Pólya's question for real entire functions, it only remains, in view of the results of Levin and Ostrovskii, to study real entire functions of infinite order and "moderate" growth( $\left.{ }^{4}\right)$.

We conclude this introduction by remarking that some striking conjectures concerning the zeros of successive derivatives of entire functions posed by Pólya in [15] remain open. R. P. Boas, Jr., brings this subject matter up-to-date in Volume II of his recent editions [5] of Pólya's papers. In view of the extensive successes in classical value distribution theory since Pólya's survey and of new representation theorems for classes of meromorphic functions resulting from these and other investigations in function theory, the time may be ripe for a reinvestigation of Pólya's problems on zero sets.

(4) Actually, Levin and Ostrovskirs result [11, Theorem 2] does not require that the zeros of $f^{\prime}$ be real. If this assumption were dropped, the Hypothetical Theorem is probably still true and would then be the analogue of Wiman's conjecture for the case of infinite order. 
2. Preliminaries. Let $f$ be a (constant multiple of a) real entire function with only real zeros. The key ideas in the proof of Theorem 1 are two representations for the logarithmic derivative $f^{\prime} / f$. Since these representations (equation (2.5) and Lemma 3 below) may be found in [10] and [11], we shall simply state the relevant lemmas.

First observe that there is no loss in generality in assuming that $f(0)$ $\neq 0$ and $f^{\prime}(0) \neq 0(5)$. Indeed if $f(0)=0$ or $f^{\prime}(0)=0$, then we consider $h(z)=f(z+\varepsilon)$ where $\varepsilon>0$ is chosen so that $f(\varepsilon) \neq 0$ and $f^{\prime}(\varepsilon) \neq 0$. It is then clear that $h(z) \in U_{2 p}$, that $h(0) \neq 0$ and $h^{\prime}(0) \neq 0$, and that $h^{(k)}(z)$ and $f^{(k)}(z)$ have the same number of complex zeros $k=1,2$. Hence, in the rest of the paper we will assume that $f(0) \neq 0$ and $f^{\prime}(0) \neq 0$.

Denote by $a_{n}$ the distinct zeros of $f$ and enumerate them as follows:

$$
\begin{aligned}
\cdots<a_{k-1}<a_{k}<a_{k+1} & <\cdots \\
& (-\infty \leqslant \alpha \leqslant k \leqslant \omega \leqslant+\infty, k \text { finite }) .
\end{aligned}
$$

According to Rolle's theorem, $f^{\prime}$ has at least one zero in each interval $\left(a_{k}, a_{k+1}\right)$; choose exactly one and denote it by $b_{k}$. We will order the $a_{k}$ 's and $b_{k}$ 's so that we have not only

$$
a_{k}<b_{k}<a_{k+1} \text { for all } k
$$

but also

$$
b_{-1}<0<a_{1}
$$

Now set( $(6)$

$$
\psi(z)=\left\{\begin{array}{l}
\frac{z-b_{0}}{z-a_{0}} \prod_{k \neq 0}\left(\frac{1-z / b_{k}}{1-z / a_{k}}\right) \text { if } \omega=+\infty, \\
\frac{z-b_{0}}{\left(z-a_{0}\right)\left(a_{\omega}-z\right)} \prod_{k \neq 0, \omega}\left(\frac{1-z / b_{k}}{1-z / a_{k}}\right) \text { if } \omega<+\infty .
\end{array}\right.
$$

We have

LEMMA 1. $\psi(z)$ maps $\operatorname{Im} z>0$ into the upper half plane.

The proof can be found in [10, pp. 308-309].

Now it is clear that the logarithmic derivative of $f$ may be represented in the form

$$
f^{\prime}(z) / f(z)=\phi(z) \psi(z)
$$

(9) The reason for having $f^{\prime}(0) \neq 0$ will become clear in the sequel.

(๑) If $f$ has no zeros, set $\psi(z)=1$; if $f$ has one zero $a_{0}$, set $\psi(z)=\left(z-a_{0}\right)^{-1}$. 
where $\phi(z)$ is an entire function. In fact we have

LEMMA 2. If $f$ is of finite order, $\phi(z)$ is a polynomial.

The proof depends on some standard estimates from Nevanlinna's theory of meromorphic functions, in particular Nevanlinna's lemma on the logarithmic derivative, and uses Carathéodory's estimate on functions mapping a half plane into a half plane. We refer the reader to [11, pp. 335-336].

Thus combining Lemmas 1 and 2 we can write, for $f \in U_{2 p}$,

$$
f^{\prime}(z) / f(z)=P(z) \psi(z)
$$

where $\psi(z)$ is given by $(2.4)$ and $P(z)$ is a polynomial. We shall call the zeros of $P$ extraordinary zeros of $f^{\prime}$ and shall call the other zeros of $f^{\prime}-$ those $b_{k}$ whose multiplicity equals 1 and those $a_{k}$ whose multiplicity exceeds 1 -ordinary zeros of $f^{\prime}$.

We remark in passing that an old result of Laguerre [6, p. 37] shows that $\operatorname{dg} P(z) \leqslant$ genus $f \leqslant 2 p+2$.

The next lemma gives another representation for $\psi(z)$.

LEMMA 3. We can write

$$
\psi(z)=\gamma z+\delta+\sum_{k=\alpha}^{\omega} A_{k}\left(\frac{1}{a_{k}-z}-\frac{1}{a_{k}}\right)
$$

where $\gamma \geqslant 0, \delta$ is real, $A_{k}>0$, and where the series $\sum_{k=\alpha}^{\omega} A_{k} / a_{k}^{2}$ converges.

The proof of this lemma can be found in [10, pp. 310-311].

Before turning to the proofs of Theorems 1 and 2 we need some technical lemmas on the growth of entire functions of finite order. The first, Lemma 4, is necessary for the proof of Theorems 1 and 2 and the other two are necessary for the proof of Theorem 2 . The proofs are by standard growth arguments and will be omitted.

LEMMA 4. Let $\Pi(z)$ be a canonical product of genus $p$, with only real zeros, let $\varepsilon>0$, and let

$$
\mathscr{D}=\left\{z=r e^{i \theta}: r>0 \text { and } \varepsilon \leqslant|\theta| \leqslant \pi-\varepsilon\right\} \text {. }
$$

Then

$$
\left|\Pi^{\prime}(z) / \Pi(z)\right|=o\left(r^{p}\right) \quad(|z|=r \rightarrow+\infty, z \in \mathbb{D})
$$

In the next two lemmas, $g(z)$ denotes an entire function of finite genus $\sigma$ with only real zeros. 
LEMMA 5. Let $g(z)$ be real with an infinite number of zeros and let $\sigma=0$ or 1 . If $N$ is a positive integer, then there exists $r_{N}$ such that

$$
|g( \pm i r)| \geqslant r^{N} \text { for } r \geqslant r_{N} \text {. }
$$

LEMMA 6. Let $g(z)$ have genus $\sigma \geqslant 2$, let $M=[\sigma / 2], \varepsilon=\pi / 2(\sigma+1)$, and $N$ be any positive integer, and let $\Phi$ be as in (2.7). Then there are at least $M$ rays, $r e^{i \psi_{1}}, \ldots, r e^{i \psi_{M}}(0<r<\infty)$ in $\mathbb{D}$ such that for $k=1, \ldots, M$,

$$
\left|g\left(r e^{i \psi_{k}}\right)\right|=o\left(r^{-N}\right) \quad(r \rightarrow \infty)
$$

3. The proof of Theorem 1 . The proof of Theorem 1 will follow fairly easily from Lemmas 7 and 8 below. In Lemma 7 we show that with at most two exceptions the extraordinary zeros of $f^{\prime \prime}$ are complex (i.e., nonreal), while in Lemma 8 we determine the number of extraordinary zeros of $f^{\prime \prime}$. Combining these lemmas we can then count the number of complex zeros of $f^{\prime \prime}$.

Assume now that $f \in U_{2 p}$. Recalling the assumptions and notations of $\$ 2$ we denote by $\left\{a_{k}\right\}$ the sequence of distinct zeros of $f$, assume that $f(0)$ $\neq 0$ and $f^{\prime}(0) \neq 0$, and write

$$
f^{\prime}(z) / f(z)=P(z) \psi(z)
$$

where $P(z)$ is the polynomial formed by the extraordinary zeros of $f^{\prime}$ and where $\psi(z)$ is as in (2.4). In view of the theorem of Pólya and Laguerre, we assume throughout this section that $p>0$; we also assume that $f^{\prime}$ has only real zeros. If we write $f=e^{Q} \Pi$ and $f^{\prime}=e^{Q_{1}} \Pi_{1}$ where $\Pi$ and $\Pi_{1}$ are the canonical products of $f$ and $f^{\prime}$ respectively, then the fact that $f^{\prime}$ has only real zeros and has only a finite number of extraordinary zeros implies that genus $\Pi=$ genus $\Pi_{1}=p_{1}$ say. Moreover, a simple growth argument shows that $\operatorname{dg}\left(Q_{1}-Q\right) \leqslant p_{1}(\leqslant 2 p+1)$. These facts easily imply that since $f \in U_{2 p}$, the same is true of $f^{\prime}$; accordingly, $f^{\prime \prime} / f^{\prime}$ has a representation similar to (3.1), say

$$
f^{\prime \prime}(z) / f^{\prime}(z)=P_{1}(z) \psi_{1}(z)
$$

where $P_{1}(z)$ is the polynomial formed by the extraordinary zeros of $f^{\prime \prime}$ and where $\psi_{1}(z)$ is the analog of $\psi(z)$ in the representation (3.1). We now state

LEMMA 7. If $f^{\prime}$ has an infinite (finite) number of zeros, then with at most one (two) exception $(s)$, the zeros of $P_{1}(z)$ are complex.

Proof. The idea behind the proof is very simple. Observe that in any finite interval determined by successive distinct zeros of $f^{\prime}, f^{\prime \prime}$ has, according to Rolle's theorem, at least one zero. Consequently, if we can show that $f^{\prime \prime}$ has at most one simple zero in every such interval and has at most one simple zero 
in the semi-infinite interval determined by a "first" or a "last" zero of $f$ ", the lemma will follow. Note then that the real zero(s) of $P_{1}(z)$ (the "exception(s)") can occur only in these semi-infinite intervals.

To be explicit, let $\left\{\gamma_{n}\right\}_{n=\alpha^{\prime}}^{\omega^{\prime}}$ denote the sequence of distinct zeros of $f^{\prime} / f$. Referring to (3.1) and (2.4), we see that this sequence is made up of the $b_{k}$ 's and the zeros of $P(z)$ (which are real in view of the assumption that $f^{\prime}$ has only real zeros). For convenience we set

$$
\gamma_{\alpha^{\prime}-1}=-\infty \text { if }-\infty<\alpha^{\prime} \text { and } \psi_{\omega^{\prime}+1}=+\infty \text { if } \omega^{\prime}<+\infty .
$$

Now observe that for any fixed $n$ either

(I) $\left(\gamma_{n}, \gamma_{n+1}\right)$ contains exactly one zero $a_{k}$ of $f$, with multiplicity $m_{k}$ or

(II) $\left(\gamma_{n}, \gamma_{n+1}\right)$ contains no zero of $f$.

Thus, we must show that in case (I) $f^{\prime \prime}$ has at most one zero in each of $\left(\gamma_{n}, a_{k}\right)$ and $\left(a_{k}, \gamma_{n+1}\right)$ if $m_{k}>1$ or at most one zero in $\left(\gamma_{n}, \gamma_{n+1}\right)$ if $m_{k}=1$, and must show that in case (II) $f^{\prime \prime}$ has at most one zero in $\left(\gamma_{n}, \gamma_{n+1}\right)$. In each case, of course, we must show that the zero is simple. We now turn to this task.

We begin by using (3.1) to write

$$
f^{\prime \prime}(z) / f^{\prime}(z)=P(z) \psi(z)+P^{\prime}(z) / P(z)+\psi^{\prime}(z) / \psi(z) .
$$

We will first deal with case (I), so that for some fixed $n$ and some zero $a_{k}$ of $f$ with multiplicity $m_{k}$ we have

$$
\gamma_{n}<a_{k}<\gamma_{n+1} .
$$

Set

$$
H_{k}(z)=\left\{\begin{array}{l}
P(z)\left(z-a_{k}\right) \psi(z)=\left(z-a_{k}\right) f^{\prime}(z) / f(z), \quad z \neq a_{k}, \\
m_{k}, \quad z=a_{k} .
\end{array}\right.
$$

In view of (3.4) then, we can write

$$
\frac{f^{\prime \prime}}{f^{\prime}}(z)=\frac{H_{k}(z)}{z-a_{k}}-\frac{1}{z-a_{k}}+\frac{H_{k}^{\prime}}{H_{k}}(z) .
$$

Now we will analyze the function $H_{k}(x)$ for $\gamma_{n}<x=\operatorname{Re} z<\gamma_{n+1}$. Assume in what follows that $\left(\gamma_{n}, \gamma_{n+1}\right)$ is a finite interval (the analysis is similar to that below in case $\left(\gamma_{n}, \gamma_{n+1}\right)$ is a semi-infinite interval). We observe that

$$
H_{k}(x) \text { is differentiable (in fact, analytic) on }\left[\gamma_{n}, \gamma_{n+1}\right]
$$

and

$$
H_{k}\left(\gamma_{n}\right)=H_{k}\left(\gamma_{n+1}\right)=0
$$

while 


$$
H_{k}(x) \neq 0 \text { on }\left(\gamma_{n}, \gamma_{n+1}\right) .
$$

Consequently, since

$$
H_{k}\left(a_{k}\right)=m_{k} \geqslant 1
$$

(3.8), (3.10) and (3.11) imply that

$$
H_{k}(x)>0 \text { for } \gamma_{n}<x<\gamma_{n+1} .
$$

Next we show that

$$
\left[H_{k}^{\prime}(x) / H_{k}(x)\right]^{\prime}<0 \text { for } \gamma_{n}<x<\gamma_{n+1} .
$$

Indeed, it follows from (3.6) and (2.4) that

$$
\begin{aligned}
\frac{H_{k}^{\prime}}{H_{k}}(x) & =\frac{P^{\prime}}{P}(x)+\frac{\psi^{\prime}}{\psi}(x)+\frac{1}{\left(x-a_{k}\right)} \\
& =\frac{P^{\prime}}{P}(x)+\sum_{j=\alpha ; j \neq k}^{\infty}\left[\frac{1}{x-b_{j}}-\frac{1}{x-a_{j}}\right]+\frac{1}{x-b_{k}} .
\end{aligned}
$$

Hence,

$$
\begin{aligned}
{\left[\frac{H_{k}^{\prime}}{H_{k}}(x)\right]^{\prime}=} & {\left[\frac{P^{\prime}}{P}(x)\right]^{\prime} } \\
& +\sum_{j=\alpha ; j \neq k}^{\omega}\left[\frac{1}{\left(x-a_{j}\right)^{2}}-\frac{1}{\left(x-b_{j}\right)^{2}}\right]-\frac{1}{\left(x-b_{k}\right)^{2}} .
\end{aligned}
$$

Examining the right-hand side of (3.15) we see that, on the one hand,

$$
\left[P^{\prime}(x) / P(x)\right]^{\prime}<0 \text { for } \gamma_{n}<x<\gamma_{n+1}
$$

since $P$ is a polynomial with real zeros, none of which lie in $\left(\gamma_{n}, \gamma_{n+1}\right)$. On the other hand, (2.2) and (3.5) and the nature of the $\gamma$ 's, imply that

$$
\cdots<a_{k-1}<b_{k-1} \leqslant \gamma_{n}<a_{k}<\gamma_{n+1} \leqslant b_{k}<a_{k+1}<\cdots ;
$$

hence, for $\gamma_{n}<x<\gamma_{n+1}$ we have

$$
\left|x-b_{j}\right|<\left|x-a_{j+1}\right| \text { for } j \geqslant k
$$

and

$$
\left|x-b_{j}\right|<\left|x-a_{j}\right| \text { for } j<k .
$$

Thus it follows from (3.18) and (3.19) that 


$$
\begin{aligned}
\sum_{j \neq k}\left[\frac{1}{\left(x-a_{j}\right)^{2}}\right. & \left.-\frac{1}{\left(x-b_{j}\right)^{2}}\right]-\frac{1}{\left(x-b_{k}\right)^{2}} \\
= & \sum_{j \geqslant k}\left[\frac{1}{\left(x-a_{j+1}\right)^{2}}-\frac{1}{\left(x-b_{j}\right)^{2}}\right] \\
& +\sum_{j<k}\left[\frac{1}{\left(x-a_{j}\right)^{2}}-\frac{1}{\left(x-b_{j}\right)^{2}}\right]<0 .
\end{aligned}
$$

Inequality (3.13) now follows from (3.15), (3.16) and (3.20).

Now we observe that (3.13) implies that $H_{k}^{\prime}(x)$ has exactly one zero on the interval $\left(\gamma_{n}, \gamma_{n+1}\right)$. Indeed (3.14) and our assumption that $\left(\gamma_{n}, \gamma_{n+1}\right)$ is a finite interval imply that

$$
\lim _{x \rightarrow \gamma_{n}^{+}} \frac{H_{k}^{\prime}}{H_{k}}(x)=+\infty, \quad \lim _{x \rightarrow \gamma_{n+1}^{-}} \frac{H_{k}^{\prime}}{H_{k}}(x)=-\infty .
$$

Since $\left[H_{k}^{\prime}(x) / H_{k}\right]^{\prime}<0$ on $\left(\gamma_{n}, \gamma_{n+1}\right),(3.21)$ implies that $H_{k}^{\prime}(x) / H_{k}$ assumes all values exactly once on this interval; in particular,

$$
H_{k}^{\prime}(x) \text { has exactly one zero on }\left(\gamma_{n}, \gamma_{n+1}\right) \text {, say at } x_{0} \text {. }
$$

Then (3.9), (3.12) and (3.22) imply that

$$
H_{k}^{\prime}(x) \begin{cases}>0 & \text { for } \gamma_{n}<x<x_{0} \\ =0 & \text { for } x=x_{0} \\ <0 & \text { for } x_{0}<x<\gamma_{n+1} .\end{cases}
$$

Moreover, (3.12) and (3.23) imply that

$$
\frac{H_{k}^{\prime}}{H_{k}}(x) \begin{cases}>0 & \text { for } \gamma_{n}<x<x_{0} \\ =0 & \text { for } x=x_{0} \\ <0 & \text { for } x_{0}<x<\gamma_{n+1}\end{cases}
$$

Now suppose that

$$
\gamma_{n}<x_{0}<a_{k}
$$

(In case $a_{k}<x_{0}<\gamma_{n+1}$ or $x_{0}=a_{k}$, the analysis is similar to that below.) Setting $z=x$ in (3.7), multiplying by $x-a_{k}$, and differentiating, we obtain

$$
\left[\left(x-a_{k}\right) \frac{f^{\prime \prime}}{f^{\prime}}(x)\right]^{\prime}=H_{k}^{\prime}(x)+\left(x-a_{k}\right)\left[\frac{H_{k}^{\prime}}{H_{k}}(x)\right]^{\prime}+\frac{H_{k}^{\prime}}{H_{k}}(x) .
$$

In view of (3.13) and (3.23)-(3.25), it follows from (3.26) that 


$$
\left[\left(x-a_{k}\right) f^{\prime \prime}(x) / f^{\prime}(x)\right]^{\prime}>0 \text { for } \gamma_{n}<x \leqslant x_{0} .
$$

In addition (3.11) and (3.23) imply

$$
H_{k}(x)>H_{k}\left(a_{k}\right)=m_{k} \geqslant 1 \text { for } x_{0} \leqslant x<a_{k} .
$$

Hence it follows from (3.7), (3.24), (3.25) and (3.28) that

$$
\left(x-a_{k}\right) \frac{f^{\prime \prime}}{f^{\prime}}(x)=H_{k}(x)-1+\left(x-a_{k}\right) \frac{H_{k}^{\prime}}{H_{k}}(x) \geqslant H_{k}(x)-1>0
$$

for $x_{0} \leqslant x<a_{k}$. On the other hand, (3.13) and (3.23)-(3.26) imply that

$$
\left[\left(x-a_{k}\right) f^{\prime \prime}(x) / f^{\prime}(x)\right]^{\prime}<0 \text { for } a_{k}<x<\gamma_{n+1} \text { and } m_{k}>1 \text {. }
$$

If $m_{k}=1$, then $f^{\prime}\left(a_{k}\right) \neq 0$ so that $\left(x-a_{k}\right) f^{\prime \prime}(x) / f^{\prime}(x)$ is differentiable at $x=a_{k}$; thus,

$$
\left[\left(x-a_{k}\right) f^{\prime \prime}(x) / f^{\prime}(x)\right]^{\prime}<0 \text { for } a_{k} \leqslant x<\gamma_{n+1} \text { and } m_{k}=1 .
$$

Now if $m_{k}>1$, it follows from (3.27) and (3.29) that $f^{\prime \prime}$ has at most one simple zero on $\left(\gamma_{n}, a_{k}\right)$, and it follows from (3.30) that $f^{\prime \prime}$ has at most one simple zero on $\left(a_{k}, \gamma_{n+1}\right)$. If, on the other hand, $m_{k}=1,(3.27)$ implies that $f^{\prime \prime}$ has at most one simple zero on $\left(\gamma_{n}, x_{0}\right]$, while (3.29) and (3.31) imply that $f^{\prime \prime}$ has no zeros on $\left(x_{0}, \gamma_{n+1}\right)$; hence, if $m_{k}=1, f^{\prime \prime}$ has at most one simple zero on $\left(\gamma_{n}, \gamma_{n+1}\right)$. This completes the proof in case (I).

We turn now to case (II). Here we assume that the interval $\left(\gamma_{n}, \gamma_{n+1}\right)$ contains no zero of $f$; i.e., no $a_{k}\left({ }^{7}\right)$. Since the proof in this case is quite similar to the proof in case (I), the reader may want to proceed directly to Lemma 8.

Here we set

$$
H(z)=P(z) \cdot \psi(z)
$$

and rewrite (3.4) as

$$
f^{\prime \prime}(z) / f^{\prime}(z)=H(z)+H^{\prime}(z) / H(z) .
$$

As before, we analyze the function $H(x)$ on the interval $\gamma_{n}<x=\operatorname{Re} z$ $<\gamma_{n+1}$. We also assume, as before, that $\left(\gamma_{n}, \gamma_{n+1}\right)$ is a finite interval; the analysis is similar to that below in case $\left(\gamma_{n}, \gamma_{n+1}\right)$ is a semi-infinite interval.

Now

$$
\begin{gathered}
H(x) \text { is differentiable on }\left[\gamma_{n}, \gamma_{n+1}\right], \\
H\left(\gamma_{n}\right)=H\left(\gamma_{n+1}\right)=0,
\end{gathered}
$$

and

( 7 Note that this includes the situation $f(z) \neq 0$. 


$$
H(x) \neq 0 \text { for } \gamma_{n}<x<\gamma_{n+1} .
$$

Thus, (3.34) and (3.36) imply that $H(x)$ is of constant sign on $\left(\gamma_{n}, \gamma_{n+1}\right)$, say

$$
H(x)>0 \text { on }\left(\gamma_{n}, \gamma_{n+1}\right)
$$

(the analysis is similar to that below if $H(x)<0$ on $\left(\gamma_{n}, \gamma_{n+1}\right)$ ).

An analysis similar to that used on $H_{k}(x)$ in case (I) shows that

$$
\left[H^{\prime}(x) / H\right]^{\prime}<0 \text { for } \gamma_{n}<x<\gamma_{n+1}
$$

and that

$$
H^{\prime}(x) \text { has exactly one zero on }\left(\gamma_{n}, \gamma_{n+1}\right) \text {, say at } x_{0} \text {. }
$$

Thus (3.34), (3.37) and (3.39) imply that

$$
H^{\prime}(x) \begin{cases}>0 & \text { for } \gamma_{n}<x<x_{0}, \\ =0 & \text { for } x=x_{0}, \\ <0 & \text { for } x_{0}<x<\gamma_{n+1} .\end{cases}
$$

Furthermore, (3.37) and (3.39) imply that

$$
\frac{H^{\prime}}{H}(x) \begin{cases}>0 & \text { for } \gamma_{n}<x<x_{0} \\ =0 & \text { for } x=x_{0} \\ <0 & \text { for } x_{0}<x<y_{n+1}\end{cases}
$$

In view of (3.37) and (3.41) it follows from (3.33) that

$$
f^{\prime \prime}(x) / f^{\prime}(x)=H(x)+H^{\prime}(x) / H(x)>0 \text { for } \gamma_{n}<x \leqslant x_{0} .
$$

Moreover, differentiating (3.42) and using (3.38) and (3.40) it follows that

$$
\left[f^{\prime \prime}(x) / f^{\prime}(x)\right]^{\prime}=H^{\prime}(x)+\left[H^{\prime}(x) / H(x)\right]^{\prime}<0 \text { for } x_{0}<x<\gamma_{n+1} .
$$

Clearly then, (3.42) and (3.43) imply that $f^{\prime \prime}$ has at most one simple zero on $\left(\gamma_{n}, \gamma_{n+1}\right)$.

This completes the proof of Lemma 7.

In the next lemma we suppose $f \in U_{2 p}, P(z)$ is the polynomial in the representation (3.1), and we determine the number of extraordinary zeros of $f^{\prime}$; i.e., the degree of $P(z)$. In the proof of Theorem 1, we shall apply this lemma to $f^{\prime}$, which is also in $U_{2 p}$, in order to determine the number of extraordinary zeros of $f^{\prime \prime}$.

LEMMA 8. If $f$ has an infinite number of zeros, then $P(z)$ is of degree $2 p$ or $2 p+1$. If $f$ has a finite number of zeros, then $P(z)$ is of degree equal to the 
genus of $f$ (i.e., is of degree $2 p, 2 p+1$, or $2 p+2$ ).

Proof. Since $f \in U_{2 p}$, we can write

$$
f(z)=e^{Q(z)} \Pi(z)
$$

where

$$
Q(z)=-a z^{2 p+2}+b z^{2 p+1}+c z^{2 p}+\cdots, \quad a \geqslant 0,
$$

and where $\Pi(z)$ is the canonical product of the zeros of $f$ and is of genus $p_{1} \leqslant 2 p+1\left({ }^{8}\right)$. All of the coefficients of $Q$, except possibly the constant term, are real.

Now let

$$
\sigma=\operatorname{genus} f=\max \left(\operatorname{dg} Q, p_{1}\right)
$$

and observe that since $f \in U_{2 p}$,

$$
2 p \leqslant \sigma \leqslant 2 p+2
$$

with

$$
\sigma=2 p+2 \text { iff } a>0
$$

while

$$
\sigma=2 p \text { iff }\left\{\begin{array}{l}
a=b=0 \text { and } p_{1}=2 p \text { or } \\
a=b=0, c>0 \text { and } p_{1} \leqslant 2 p-1 .
\end{array}\right.
$$

If $f$ has only a finite number of zeros (i.e., if $\Pi(z)$ is a polynomial), a simple counting argument shows that

$$
\operatorname{dg} P(z)=\operatorname{dg} Q(z)\left({ }^{9}\right) \quad(=\text { genus } f) .
$$

So let us assume in what follows that $f$ has an infinite number of zeros. On the one hand, using (3.44) and (3.45) we can write

$$
\begin{aligned}
\frac{f^{\prime}}{f}(z)= & Q^{\prime}(z)+\frac{\Pi^{\prime}}{\Pi}(z) \\
= & -a(2 p+2) z^{2 p+1}+b(2 p+1) z^{2 p} \\
& +2 c p z^{2 p-1}+\cdots+z^{p_{1}} \sum_{k=\alpha}^{\omega} \frac{m_{k}}{a_{k}^{p_{1}}\left(z-a_{k}\right)}
\end{aligned}
$$

where $m_{k}=$ multiplicity of $a_{k}$. On the other hand, we can write, as in (3.1),

(8) Recall the assumption that $f(0) \neq 0$.

(9) If $P$ has no zeros $\operatorname{dg} P(z)=\operatorname{dg} Q(z)-1$. 


$$
f^{\prime}(z) / f(z)=P(z) \psi(z)
$$

where $P(z)$ is the polynomial of the extraordinary zeros of $f^{\prime}$ and where $\psi(z)$ is as in (2.4). Applying Lemma 3 to $\psi(z),(3.52)$ becomes

$$
\frac{f^{\prime}}{f}(z)=P(z)\left\{\gamma z+\delta+\sum_{k=\alpha}^{\omega} A_{k}\left(\frac{1}{a_{k}-z}-\frac{1}{a_{k}}\right)\right\}
$$

where

$$
\gamma \geqslant 0, \quad A_{k}>0, \quad \text { and } \quad \sum_{k=\alpha}^{\omega} \frac{A_{k}}{a_{k}^{2}}<\infty .
$$

Combining (3.51) and (3.53) we have,

$$
\begin{aligned}
& -a(2 p+2) z^{2 p+1}+b(2 p+1) z^{2 p}+2 c p z^{2 p-1}+\cdots \\
& \quad+z^{p_{1}} \sum_{k=\alpha}^{\omega} \frac{m_{k}}{a_{k}^{p_{1}}\left(z-a_{k}\right)}=P(z)\left\{\gamma z+\delta+\sum_{k=\alpha}^{\omega} A_{k}\left(\frac{1}{a_{k}-z}-\frac{1}{a_{k}}\right)\right\} .
\end{aligned}
$$

Equating residues at $z=a_{k}$, we get

$$
m_{k}=-A_{k} P\left(a_{k}\right), \quad \alpha \leqslant k \leqslant \omega,
$$

or

$$
P\left(a_{k}\right)=-m_{k} / A_{k}<0, \quad \alpha \leqslant k \leqslant \omega .
$$

Now, set $d=\operatorname{degree} P(z)$ and choose $M>0$ so that

$$
0<-P\left(a_{k}\right) \leqslant M\left|a_{k}\right|^{d}, \quad \alpha \leqslant k \leqslant \omega .
$$

Then (3.54) and (3.57) imply

$$
\frac{1}{M} \sum_{k} \frac{m_{k}}{\left|a_{k}\right|^{d+2}} \leqslant \sum_{k} \frac{-m_{k}}{a_{k}^{2} P\left(a_{k}\right)}=\sum_{k} \frac{A_{k}}{a_{k}^{2}}<\infty .
$$

Hence,

$$
d+2 \geqslant p_{1}+1 \text { or } d \geqslant p_{1}-1 .
$$

Let $q$ be any integer satisfying

$$
q \geqslant \max (d+1, \sigma-1) .
$$

Differentiating (3.55) $q$ times yields 


$$
\begin{aligned}
& (q+1) ! B+\frac{d^{q}}{d z^{q}}\left\{z^{p_{1}} \sum_{k} \frac{m_{k}}{a_{k}^{p_{1}}\left(z-a_{k}\right)}\right\} \\
& \quad=\frac{d^{q}}{d z^{q}}[P(z)(\gamma z+\delta)]+\sum_{k}\left[\frac{d^{q}}{d z^{q}}\left(\frac{A_{k} \cdot P(z)}{a_{k}-z}\right)-\frac{A_{k}}{a_{k}} \frac{d^{q}}{d z^{q}} P(z)\right]
\end{aligned}
$$

where, in view of (3.46)-(3.49), (3.59), and (3.60).

$$
B= \begin{cases}-a \leqslant 0 & \text { if } q=2 p+1 \\ b & \text { if } q=2 p \\ c>0 & \text { if } q=2 p-1 \\ 0 & \text { if } q>2 p+1\end{cases}
$$

Turning to the left-hand side of (3.61) for a moment, it is easy to see that

$$
z^{p_{1}} \sum_{k} \frac{1}{a_{k}^{p_{1}}\left(z-a_{k}\right)}=\sum_{k}\left[\frac{1}{z-a_{k}}+\frac{1}{a_{k}} T\left(\frac{z}{a_{k}}\right)\right]
$$

where

$$
T(z)=z^{p_{1}-1}+z^{p_{1}-2}+\cdots+z+1 .
$$

Consequently, since $q \geqslant d+1 \geqslant p_{1}$, (3.61) becomes

$$
\begin{aligned}
(q+1) ! B+ & \sum_{k} m_{k} \frac{d^{q}}{d z^{q}}\left(\frac{1}{z-a_{k}}\right) \\
& =\gamma \frac{d^{q}}{d z^{q}}[z \cdot P(z)]-\sum_{k} \frac{d^{q}}{d z^{q}}\left(\frac{A_{k} \cdot P(z)}{z-a_{k}}\right) .
\end{aligned}
$$

And, in view of (3.56), (3.64) becomes

$$
(q+1) ! B+\sum_{k} m_{k} \frac{d^{q}}{d z^{q}}\left(\frac{1}{z-a_{k}}\right)=\gamma \frac{d^{q}}{d z^{q}}[z \cdot P(z)]+\sum_{k} m_{k} \frac{d^{q}}{d z^{q}}\left(\frac{1}{z-a_{k}}\right)
$$

or

$$
(q+1) ! B=\gamma \frac{d^{q}}{d z^{q}}[z \cdot P(z)] .
$$

We will now use the fundamental relations (3.59) and (3.65) to show that if $d$ is even, then $d=2 p$ and if $d$ is odd, then $d=2 p+1$. Before doing this, we need to make a couple of observations. First, note that by (3.57),

$$
\lim _{k \rightarrow-\infty} a_{k}=-\infty \text { and } \lim _{k \rightarrow+\infty} a_{k}=+\infty \text { implies } d \text { even. }
$$

Next, 


$$
\sigma=2 p \text { implies } d \text { even. }
$$

To see this, we suppose, in view of (3.66), that $f$ has a finite number of positive zeros or perhaps none at all (the argument is similar in case $f$ has a finite number of negative zeros). Then, since $\sigma=2 p$, we have by (3.51)

$$
\frac{f^{\prime}}{f}(z)=2 c p z^{2 p-1}+\cdots+z^{p_{1}} \sum_{k=-\infty}^{\omega} \frac{m_{k}}{a_{k}^{p_{1}}\left(z-a_{k}\right)} \quad(\omega<+\infty) .
$$

Taking into account (3.49), it is easy to see that (3.68) implies

$$
\lim _{x \rightarrow a_{\omega}^{+}} \frac{f^{\prime}}{f}(x)=+\infty \text { and } \lim _{x \rightarrow+\infty} \frac{f^{\prime}}{f}(x)=+\infty
$$

where $x=\operatorname{Re} z$. Thus, on the interval $\left(a_{\omega},+\infty\right), f^{\prime}$ has an even number of zeros. This means, in view of the representation (3.52) and the nature of $\psi(z)$, that $P(z)$ has an even number of zeros on $\left(a_{\omega},+\infty\right)$. Equation (3.57) then implies that $d$ is even.

Finally, we note that

$$
d \leqslant 2 p+1 .
$$

To see this first observe that (3.51) and Lemma 4 and the fact that $\Pi(z)$ is of genus $p_{1} \leqslant 2 p+1$ imply

$$
\left|f^{\prime}(i y) / f(i y)\right| \leqslant a(2 p+2)|y|^{2 p+1}+o\left(|y|^{2 p+1}\right) \quad(|y| \rightarrow \infty)
$$

and

(3.72) $\operatorname{Im}\left[f^{\prime}(i y) / f(i y)\right]=-a(-1)^{p}(2 p+2) y^{2 p+1}+o\left(|y|^{2 p+1}\right) \quad(|y| \rightarrow \infty)$,

$a \geqslant 0$. Next recall Lemma 1, apply Carathéodory's estimate on functions mapping a half plane into a half plane $[10$, p. 18$]$ to $\psi(z)$, and use (3.1) to obtain

$$
\left|f^{\prime}(i y) / f(i y)\right| \geqslant k|y|^{d-1} \quad(k=\text { constant }>0) .
$$

Comparing this inequality to (3.71) we have

$$
d \leqslant 2 p+2 \text {. }
$$

If $d=2 p+2$, and $a=0$, a comparison of (3.71) and (3.73) yields $d$ $\leqslant 2 p+1$. Thus, if $d=2 p+2$, we also have $a>0$. But then (3.1) implies

$$
\operatorname{Im}\left[f^{\prime}(i y) / f(i y)\right] \sim k_{1}(-1)^{p+1} y^{2 p+2} \operatorname{Im} \psi(i y) \quad(|y| \rightarrow \infty)
$$


where $k_{1}=$ leading coefficient of $P<0$, and, in view of Lemma 1 , this asymptotic behavior is incompatible with that expressed by (3.72) $\left(k_{1}<0\right.$ is a consequence of (3.57) and the assumption that $d=2 p+2)$. Consequently $d=2 p+2$ is not possible and (3.70) now follows from (3.74).

We now consider separately the cases $d$ even, $d$ odd:

(I) Suppose $d$ is even. In this case we will show that $d=2 p$. Clearly, in view of (3.70) $d \leqslant 2 p$. To show that $d \geqslant 2 p$, first observe that if $p_{1} \geqslant 2 p$, then (3.59) implies that $d \geqslant 2 p-1$. Since we are assuming that $d$ is even, it follows that

$$
d \geqslant 2 p \quad\left(p_{1} \geqslant 2 p\right) .
$$

Now suppose that $p_{1} \leqslant 2 p-1$ and $d \leqslant 2 p-2$. Then, since $\sigma \geqslant 2 p$, we choose $q=\sigma-1$ in (3.60) to get

$$
(q+1) ! B=\gamma \frac{d^{q}}{d z^{q}}[z \cdot P(z)] .
$$

Moreover, since $p_{1} \leqslant 2 p-1$, (3.46) and (3.47) imply $\sigma=\operatorname{dg} Q$; hence, $B \neq 0$. Thus, we must have $q=d+1=2 p-1$ or, in view of (3.62),

$$
d=\sigma-2=2 p-2 \text { and } B=c>0 .
$$

On the other hand, by (3.57), $P\left(a_{k}\right)<0$ for all $k$, and this implies, since $d$ is even, that the leading coefficient of $P(z)$ is negative. But this contradicts (3.76) and (3.77); hence, the case $d \leqslant 2 p-2$ is not possible. Thus we have

$$
d \geqslant 2 p \quad\left(p_{1} \leqslant 2 p-1\right)
$$

and so, combining (3.75) and (3.78), have in all cases, $d \geqslant 2 p$.

(II) Suppose $d$ is odd. In this case we will show that $d=2 p+1$. In view of (3.70), we must show that $d \geqslant 2 p+1$. To this end, first note that if $p_{1} \geqslant 2 p+1$, then (3.59) implies that $d \geqslant 2 p$, and thus, since $d$ is odd,

$$
d \geqslant 2 p+1 \quad\left(p_{1} \geqslant 2 p+1\right) .
$$

Now suppose that $p_{1} \leqslant 2 p$ and $d \leqslant 2 p-1$. Since (3.67) implies $\sigma \geqslant 2 p$ +1 , we choose $q=\sigma-1$ in (3.60) and reason as above to get

$$
(q+1) ! B=\gamma \frac{d^{q}}{d z^{q}}[z \cdot P(z)], \quad B \neq 0,
$$

in fact,

$$
d=\sigma-2=2 p-1 \text { and } B=b \neq 0 .
$$


In view of (3.66) we suppose $f$ has a finite number of positive zeros (the argument is similar if $f$ has a finite number of negative zeros). Since $P\left(a_{k}\right)<0$ for all $k$, and $\operatorname{dg} P=d$ is odd, we must have the leading coefficient of $P>0$; hence, since $\gamma \geqslant 0((3.54))(3.80)$ implies $B=b>0$. So, by (3.81), (3.46), and (3.51) we have,

$$
\frac{f^{\prime}}{f}(z)=b(2 p+1) z^{2 p}+\cdots+z^{p_{1}} \sum_{k=-\infty}^{\omega} \frac{m_{k}}{a_{k}^{p_{1}}\left(z-a_{k}\right)},
$$

where now $b>0, p_{1} \leqslant 2 p, \omega<\infty$. Thus,

$$
\lim _{x \rightarrow a_{\omega}^{+}} \frac{f^{\prime}}{f}(x)=+\infty \text { and } \lim _{x \rightarrow+\infty} \frac{f^{\prime}}{f}(x)=+\infty
$$

and therefore in the interval $\left(a_{\omega},+\infty\right), f^{\prime}$ has an even number of zeros. But this implies (cf. the reasoning following equation (3.69)), $P(z)$ is of even degree. This contradicts the assumption that $d$ is odd; hence, the case $d \leqslant 2 p-1$ is not possible and we have

$$
d \geqslant 2 p+1 \quad\left(p_{1} \leqslant 2 p\right)
$$

Combining (3.79) and (3.82) we have, in all cases, $d \geqslant 2 p+1$.

The proof of Lemma 8 is now complete.

Proof of Theorem 1. Let $f \in U_{2 p}$. By virtue of the remarks made at the beginning of this section, we have $f^{\prime} \in U_{2 p}$ and so, by (3.2),

$$
\frac{f^{\prime \prime}}{f^{\prime}}(z)=P_{1}(z) \psi_{1}(z)
$$

Thus the number of complex zeros of $f^{\prime \prime}$ equals the number of complex zeros of $P_{1}(z)$ (this of course is an even number since $f^{\prime \prime}$ is a constant multiple of a real function).

If $f^{\prime}$ has an infinite number of zeros, then by Lemma 8, applied to $f^{\prime}, P_{1}(z)$ has $2 p$ or $2 p+1$ zeros; moreover, by Lemma 7 , at most one of these is real. Hence, $f^{\prime \prime}$ has $2 p$ complex zeros.

If $f^{\prime}$ has a finite number of zeros, then by Lemma $8, P_{1}(z)$ has $2 p, 2 p+1$, or $2 p+2$ zeros, and by Lemma 7 , at most two of these are real. Hence, if $\operatorname{dg} P_{1}(z)=2 p+1$, then $f^{\prime \prime}$ has $2 p$ complex zeros.

On the other hand, if $d_{1}=\operatorname{dg} P_{1}(z)=2 p+2$ or $2 p$ a slightly more detailed analysis is needed. We write

$$
f^{\prime}(z)=e^{Q_{1}(z)} \prod_{k=\alpha}^{\omega}\left(1-\frac{z}{c_{k}}\right)^{m_{k}}
$$

where 


$$
Q_{1}(z)=-a^{\prime} z^{2 p+2}+b^{\prime} z^{2 p+1}+c^{\prime} z^{2 p}+\cdots, \quad a^{\prime} \geqslant 0,
$$

and where $c_{k},-\infty<\alpha \leqslant k \leqslant \omega<+\infty$, is a zero of $f^{\prime}$ of multiplicity $m_{k}$. If $\sigma_{1}$ denotes the genus of $f^{\prime}$, then applying Lemma 8 to $f^{\prime}$ we have $\sigma_{1}=\operatorname{dg} Q_{1}(z)$ $=d_{1}=2 p+2$ or $2 p$. Further, equations (3.48) and (3.49), modified for $f^{\prime}$, imply

$$
\begin{cases}a^{\prime}>0 & \text { if } d_{1}=2 p+2 \\ a^{\prime}=b^{\prime}=0, \quad c^{\prime}>0 & \text { if } d_{1}=2 p\end{cases}
$$

From (3.83) we obtain

$$
\begin{aligned}
\frac{f^{\prime \prime}}{f^{\prime}}(x)= & -a^{\prime}(2 p+2) x^{2 p+1}+b^{\prime}(2 p+1) x^{2 p} \\
& +2 c^{\prime} p x^{2 p-1}+\cdots+\sum_{k=\alpha}^{\omega} \frac{m_{k}}{x-c_{k}}
\end{aligned}
$$

hence,

$$
\lim _{x \rightarrow c_{\alpha}^{-}} \frac{f^{\prime \prime}}{f^{\prime}}(x)=-\infty
$$

and, in view of (3.84),

$$
\lim _{x \rightarrow-\infty} \frac{f^{\prime \prime}}{f^{\prime}}(x)= \begin{cases}+\infty & \text { if } d_{1}=2 p+2, \\ -\infty & \text { if } d_{1}=2 p\end{cases}
$$

Thus on the interval $\left(-\infty, c_{\alpha}\right), f^{\prime \prime}$ has an odd (even) number of zeros if $d_{1}=2 p+2(2 p)$. A similar analysis on $\left(c_{\omega},+\infty\right)$ yields the same conclusion. But by Lemma 7 and the initial remarks of its proof $f^{\prime \prime}$ has at most one zero on each of these intervals; hence, $f^{\prime \prime}$ has one (no) zero in each of $\left(-\infty, c_{\alpha}\right)$ and $\left(c_{\omega},+\infty\right)$ if $d_{1}=2 p+2(2 p)$. Thus, since the real zeros of $P_{1}(z)$ can occur only in these semi-infinite intervals, it follows that in either case $d_{1}=2 p+2$ or $2 p, f^{\prime \prime}$ has $2 p$ complex zeros.

4. The proof of Theorem 2. In what follows we shall assume that $f$ is an entire function of finite order and that $f$ and $f^{\prime}$ have only real zeros. In addition, in view of Corollary 1, we shall assume that $f$ is not a constant multiple of a real function.

In the case that $f$ has a finite number of zeros, we shall show that these assumptions together with the assumption that $f^{\prime \prime}$ also has only real zeros imply that

$$
f(z)=a e^{b z} \quad(a, b \text { constants, } b \text { complex })
$$


In the case that $f$ has an infinite number of zeros, we shall show that these assumptions imply that either

$$
f(z)=a\left(e^{i c z}-e^{i d}\right) \quad(a, c, d \text { constants, } c, d \text { real })
$$

or $f^{\prime \prime}$ has an infinite number of complex zeros. Consequently, the only entire functions satisfying the hypothesis of Theorem 2 are those $f \in U_{0}$ or those $f$ of the form (4.1) or (4.2).

Our proof essentially follows Alander's very sketchy outline [4]. In addition, we remark that Pólya [14] has showed that if $f$ has a finite number of (real) zeros and $f^{\prime}$ and $f^{\prime \prime}$ have only real zeros, then $f$ is of the form (4.1) or is a constant multiple of a real function. We will not make use of his result however.

Assuming now that $f$ is not (a constant multiple of) a real function, we can write

$$
f(z)=e^{i Q(z)} f_{1}(z)
$$

where $Q(z)$ is a real polynomial of degree $q \geqslant 1$ and $f_{1}(z)$ is a real entire function of finite order with only real zeros. Taking the logarithmic derivative of $f$, we have

$$
f^{\prime}(z) / f(z)=i Q^{\prime}(z)+f_{1}^{\prime}(z) / f_{1}(z) .
$$

Since $Q(z)$ and $f_{1}(z)$ are real, it now follows that, aside from multiple zeros of $f, f^{\prime}$ has at most $q-1$ real zeros, each of which is a zero of $Q^{\prime}(z)$. Consequently, since $f^{\prime}$ has only real zeros, we can write

$$
f^{\prime}(z) / f(z)=p(z) / g(z)
$$

where $p(z)$ is a real polynomial determined by those real zeros of $f^{\prime}$ which are distinct from multiple zeros of $f$ and where $g(z)$ is a transcendental entire function of finite order with only real zeros. Note also that by our remark above, each zero of $p(z)$ is also a zero of $Q^{\prime}(z)$; i.e., $p(z)$ divides $Q^{\prime}(z)$.

Now we use Lemma 4 and Lemma 6 to estimate the growth of $g$. Write

$$
f_{1}(z)=e^{Q_{1}(z)} \Pi_{1}(z)
$$

where $Q_{1}(z)$ is a real polynomial of degree $q_{1}$ and $\Pi_{1}(z)$ is the canonical product of the zeros of $f_{1}$ of genus $p_{1}$, say (here we assume, as in the proof of Theorem 1 , that $f(0) \neq 0)$. If $\sigma=$ genus $g, \varepsilon=\pi / 2(\sigma+1)$, and $\Phi$ is as in (2.7), then by Lemma 4 , we have 


$$
\begin{aligned}
\left|f_{1}^{\prime}(z) / f_{1}(z)\right| & \leqslant\left|Q_{1}^{\prime}(z)\right|+\left|\Pi_{1}^{\prime}(z) / \Pi_{1}\right| \\
& =O\left(r^{q_{1}-1}\right)+o\left(r^{p_{1}}\right) \quad(|z|=r \rightarrow \infty, z \in \text { D) } .
\end{aligned}
$$

Thus on the one hand, (4.4) and (4.7) imply that

$$
\left|f^{\prime}(z) / f(z)\right|=O\left(r^{k}\right) \quad(|z|=r \rightarrow \infty, z \in \mathscr{D})
$$

where $k=\max \left(q-1, q_{1}-1, p_{1}\right)$.

On the other hand, if we set $N=k+1$, an application of Lemma 6 to (4.5) shows that if $g(z)$ has genus $\sigma \geqslant 2$, then there are $[\sigma / 2]$ rays $r e^{i \psi j} \in \mathbb{D}$ such that

$$
\left|f^{\prime}\left(r e^{i \psi_{j}}\right) / f\left(r e^{i \psi_{j}}\right)\right| \geqslant r^{k+1} \quad\left(r \geqslant r_{0}, j=1,2, \ldots,[\sigma / 2]\right) .
$$

This is incompatible with (4.8); consequently, $g(z)$ must be of genus $\leqslant 1$.

Suppose then that $g(z)=e^{i(\alpha z+\beta)} g_{0}(z)$ where $\alpha$ and $\beta$ are real constants and $g_{0}(z)$ is a real entire function of genus $\leqslant 1$ with only real zeros. By (4.5), we have

$$
\frac{f^{\prime}}{f}(z)=\frac{p(z)}{e^{i(\alpha z+\beta)} g_{0}(z)}=\frac{p(z)}{g_{0}(z)}[\cos (\alpha z+\beta)-i \sin (\alpha z+\beta)] .
$$

Since the functions $Q^{\prime}(z)$ and $f_{1}^{\prime}(z) / f_{1}(z)$ in (4.4) are real and the functions $p(z)$ and $g_{0}(z)$ in (4.10) are also real, it follows that for $z$ real, $g_{0}(z) \neq 0$,

$$
Q^{\prime}(z)=-\frac{p(z)}{g_{0}(z)} \sin (\alpha z+\beta)
$$

and thus

$$
\frac{1}{g_{0}(z)}=-\frac{Q^{\prime}(z)}{p(z)} \frac{1}{\sin (\alpha z+\beta)}
$$

for all $z, g_{0}(z) \neq 0$. (Note that since $p$ divides $Q^{\prime}$, it follows from (4.12) that $-Q^{\prime}(z) / p(z)$ is a real polynomial determined by a finite number of zeros of $\sin (\alpha z+\beta)$ if $\alpha \neq 0$ and is a real constant if $\alpha=0$.) Using (4.12) in (4.10) we obtain

$$
\frac{f^{\prime}}{f}(z)=\frac{-Q^{\prime}(z)}{e^{i(\alpha z+\beta)} \sin (\alpha z+\beta)}=\frac{P(z)}{e^{i(\alpha z+\beta)} \sin (\alpha z+\beta)}
$$

where $P(z)=-Q^{\prime}(z)$.

If $f$ has only a finite number of zeros, then $\alpha=0, \beta \neq 0(\bmod \pi)$, and so 


$$
\frac{f^{\prime}}{f}(z)=\frac{P(z)}{e^{i \beta} \sin \beta}=\frac{P(z)}{k}
$$

where $k=e^{i \beta} \sin \beta=$ nonreal constant.

If $f$ has an infinite number of zeros, then $\alpha \neq 0$ and it follows easily from (4.13) that

$$
\frac{f^{\prime \prime}}{f^{\prime}}(z)=\frac{[P(z)-\alpha] \cos (\alpha z+\beta)}{\sin (\alpha z+\beta)}-i[P(z)+\alpha]+\frac{P^{\prime}}{P}(z) ;
$$

hence, for $z=x+i y$ and $\alpha>0$,

$$
\begin{array}{ll}
\frac{f^{\prime \prime}}{f^{\prime}}(i y)=-2 i(1+o(1)) P(i y), & y \rightarrow+\infty, \\
\frac{f^{\prime \prime}}{f^{\prime}}(i y)=-2 i \alpha(1+o(1)), & y \rightarrow-\infty .
\end{array}
$$

In case $\alpha<0$ these limits are interchanged.

Now if $f$ has a finite number of zeros, we will use (4.14) together with the assumption that $f^{\prime \prime}$ has only real zeros to show that $f$ is of the form (4.1). On the other hand, if $f$ has an infinite number of zeros, we will use (4.16) to show that either $f$ is of the form (4.2) or $f^{\prime \prime}$ has an infinite number of complex zeros.

Turning to the first situation, we have not only (4.14), but also, since $f^{\prime}$ has a finite number of zeros and $f^{\prime \prime}$ has only real zeros,

$$
\frac{f^{\prime \prime}}{f^{\prime}}(z)=\frac{P_{1}(z)}{e^{i \beta_{1}} \sin \beta_{1}}=\frac{P_{1}(z)}{k_{1}}
$$

where $P_{1}(z)$ is a real polynomial, $\beta_{1}$ is a real constant, $\beta_{1} \neq 0(\bmod \pi)$, and $k_{1}=e^{i \beta_{1}} \sin \beta_{1}$. On the other hand, it follows from (4.14) that

$$
f^{\prime \prime}(z) / f^{\prime}(z)=P(z) / k+P^{\prime}(z) / P(z) .
$$

Since $P(z)$ is a real polynomial, (4.17) and (4.18) imply

$$
P(z)=k_{2}=\text { real constant }
$$

Hence, by (4.14),

$$
f^{\prime}(z) / f(z)=k_{2} / k=\text { nonreal constant }
$$

and thus $f$ is of the form (4.1).

Turning now to the situation where $f$ has an infinite number of zeros, we show that either $f$ is of the form (4.2) or $f^{\prime \prime}$ has an infinite number of complex zeros. To that end, suppose that $f^{\prime}$ has only a finite number of (real) zeros. Then 


$$
f^{\prime}(z)=G(z) e^{H(z)}
$$

where $G(z)$ and $H(z)$ are polynomials, $G(z)$ with only real zeros. Thus,

$$
f^{\prime \prime}(z) / f^{\prime}(z)=H^{\prime}(z)+G^{\prime}(z) / G(z)
$$

and

$$
f^{\prime \prime}(i y) / f^{\prime}(i y)=(1+o(1)) H^{\prime}(i y) \quad(|y| \rightarrow+\infty) .
$$

Comparing (4.23) to (4.16) we conclude first that

$$
H^{\prime}(z)=-2 i \alpha
$$

and second that

$$
P(z) \equiv \alpha .
$$

In view of (4.25), (4.13) then becomes

$$
\frac{f^{\prime}}{f}(z)=\frac{\alpha}{e^{i(\alpha z+\beta)} \sin (\alpha z+\beta)}=\frac{2 i \alpha}{e^{i \beta}\left(e^{2 i \alpha z} e^{i \beta}-e^{-i \beta}\right)} ;
$$

consequently,

$$
f(z)=a\left(e^{-2 i \alpha z}-e^{2 i \beta}\right), \quad a=\text { constant } .
$$

Thus, if $f^{\prime}$ has only a finite number of zeros, $f$ must be of the form (4.2).

We conclude then, that if $f$ is not of the form (4.2), $f^{\prime}$ has an infinite number of (real) zeros. In that case, we can apply to $f^{\prime}$ the reasoning of the first part of this section to deduce that, aside from multiple zeros of $f^{\prime}, f^{\prime \prime}$ has only a finite number of real zeros. If, in addition, $f^{\prime \prime}$ has only a finite number of complex zeros, then we can write

$$
f^{\prime \prime}(z) / f^{\prime}(z)=p_{1}(z) / g_{1}(z)
$$

where $p_{1}(z)$ is a polynomial and $g_{1}(z)$ is a transcendental entire function of finite genus. In fact, reasoning along the lines which lead from (4.5) to (4.9) we conclude that $g_{1}(z)$ has genus $\leqslant 1$.

Write

$$
g_{1}(z)=e^{i\left(\alpha_{1} z+\beta_{1}\right)} g_{2}(z)
$$

where $\alpha_{1}$ and $\beta_{1}$ are real constants and $g_{2}(z)$ is a real entire function of genus 0 or 1 with an infinite number of zeros. Setting $N=\operatorname{dg} p_{1}(z)+1=P_{1}+1$, it follows from Lemma 5 that for $|y|$ sufficiently large, 


$$
\left|g_{1}(i y)\right|= \begin{cases}\left|g_{2}(i y)\right| \geqslant|y|^{P_{1}+1} & \text { if } \alpha_{1}=0, \\ e^{-\alpha_{1} y}\left|g_{2}(i y)\right| \geqslant e^{-\alpha_{1} y}|y|^{P_{1}+1} & \text { if } \alpha_{1} \neq 0 .\end{cases}
$$

Hence, it now follows from (4.28) and (4.30) that

$$
\left|f^{\prime \prime}(i y) / f^{\prime}(i y)\right|=o(1)
$$

along either the positive or negative imaginary axis. This clearly contradicts (4.16).

Thus our supposition that $f^{\prime \prime}$ has at most a finite number of complex zeros is false; consequently, $f^{\prime \prime}$ has an infinite number of complex zeros. In view of our remarks at the beginning of this section, this completes the proof of Theorem 2.

\section{REFERENCES}

1. M. Allander, Sur le déplacement des zéros des fonctions entières par leur dérivation, Thèse, Uppsala, 1914.

2. - Sur les zéros extraordinaires des dérivées des fonctions entières réelles, Ark. Mat. Astronom. Fys. 11 (1916), no. 15.

3. - Sur les zéros complexes des dérivées des fonctions entières réelles, Ark. Mat. Astronom. Fys. 16 (1922), no. 10.

4. - Sur les fonctions entières qui ont tous leurs zéros sur une droit, C. R. Acad. Sci. Paris 176 (1923), 158-161.

5. R. P. Boas, Jr., George Pólya: Collected papers, Vols. I, II, MIT Press, Cambridge, Mass., 1974.

6. E. Borel, Lef̧ons sur les fonctions entières, Gauthier-Villars, Paris, 1900.

7. A. Edrei, Meromorphic functions with three radially distributed values, Trans. Amer. Math. Soc. 78 (1955), 276-293. MR 16, 808.

8. S. Hellerstein and J. Williamson, Derivatives of entire functions and a question of Polya, Bull. Amer. Math. Soc. 81 (1975), 453-455. MR 50 \# 13518.

9. E. Laguerre, Sur les fonctions du genre zéro et du genre un, C. R. Acad. Sci. 98 (1882); Oeuvres 1 (1898), 174-177.

10. B. Ja. Levin, Distribution of zeros of entire functions, GITTL, Moscow, 1956; English transl., Transl. Math. Monographs, vol. 5, Amer. Math. Soc., Providence, R.I., 1964. MR 19, 402; 28 \#217.

11. B. Ja. Levin and I. V. Ostrovskii, On the ciependence of the growth of an entire function on the distribution of the zeros of its derivatives (On a question of G. Pollya and A. Wiman), Sibirsk.Mat. Ż. 1 (1960), 427-455; English transl., Amer. Math. Soc. Transl. (2) 32 (1963), pp. 323-357. MR 24 \# A833.

12. G. Pólya, Uber Annaherung durch Polynome mit lauter reëlen Wurzeln, Rend. Circ. Mat. Palermo 36 (1913), 279-295.

13. - Sur une question concernant les fonctions entières, C. R. Acad. Sci. Paris 158 (1914), $330-333$.

14. - Bemerkung zur Theorie der ganzen Funktionen, Jber. Deutsch. Math.-Verein. 24 (1915), 392-400.

15. - On the zeros of the derivatives of a function and its analytic character, Bull. Amer. Math. Soc. 49 (1943), 178-191. MR 4, 192.

Department of Mathematics, University of Wisconsin, Madison, Wisconsin 53706

Department of Mathematics, University of Hawail, Honolulu, Hawail 96822 